\title{
Nonlinear response of antiferromagnetic films to radiation at oblique incidence
}

\author{
N. S. Almeida and A. S. Carriço \\ Departamento de Física Teórica e Experimental/Centro de Ciências Exatas, Universidade Federal do Rio Grande do Norte, \\ 59 072-970 Natal, Rio Grande do Norte, Brazil \\ (Received 5 October 1992; revised manuscript received 15 June 1993)
}

\begin{abstract}
The response of an antiferromagnetic film to incident radiation at oblique incidence is studied in the nonlinear regime. For given values of the frequency and incidence angle, the reflectivity of a film of arbitrary thickness is obtained as an implicit function of the incident field amplitude. The compound effect of the incidence angle and field strength on the reflected radiation is studied by means of the analysis of the reflection coefficient calculated for different values of these external variables. We use the physical parameters of the uniaxial Heisenberg antiferromagnet $\mathrm{FeF}_{2}$ to obtain numerical results. It is found that the threshold for nonlinear optical behavior is strongly dependent on the incidence angle.
\end{abstract}

In recent years the study of materials with nonlinear optical behavior has attracted a great deal of research effort. $^{1-12}$ The leading reason for this is the possibility of observing nonlinear effects even for modest incident radiation power. Nevertheless, for any real system, the interpretation of nonlinear behavior is not a trivial matter. However, the comprehension of the main features becomes a little easier if the system under investigation has a precise and good equilibrium structure.

In this respect, uniaxial antiferromagnets are candidates to give a good insight of these effects since they are well studied since a long time ago. In particular, the lowest-order nonlinear contribution to the dynamical susceptibility of these magnetic systems was calculated by Almeida and Mills ${ }^{5}$ some years ago. It was shown that this nonlinear correction diverges as the inverse fourth power of the frequency, measured from the resonance frequency. These authors presented numerical results for $\mathrm{FeF}_{2}$ thin films, showing that, for incident radiation frequencies close to the antiferromagnetic resonance frequency, the transmissivity of radiation at normal incidence is strongly dependent on the incident power. Furthermore it was predicted that the nonlinear optical multistability should be observed for incident power available in many laboratories. Also, the nonlinear susceptibility ${ }^{5}$ was later used to investigate the transmissivity of antiferromagnetic superlattices in the nonlinear regime. ${ }^{6,7}$

Recently a study of radiation transmission through nonlinear dielectric films at oblique incidence ${ }^{8}$ was reported, extending previous results for normal incidence. ${ }^{9}$ These studies focused on the effects introduced by the frequency independent nonlinear part of the dielectric function on the optical properties of the films. Particular attention was given to the regime of total reflection. It was shown that threshold for total reflection in thin films should be modified by the nonlinear part of the dielectric function.

So far the theoretical investigation of nonlinear optical properties of antiferromagnetic films and superlattices has been restricted to the normal incidence case. The extension to oblique incidence adds considerable insight into the optical properties of these films. In a certain sense this corresponds to varying the film thickness.

Under the nonlinear regime, it is not straightforward to predict the optical properties, because the refractive index depends upon the radiation frequency and power. It has been argued ${ }^{10}$ that the effective refractive index of antiferromagnetic films in the nonlinear regime is a functional of the magnetic field, involving its value throughout the whole film. In other words, the refractive index is a global function of the field inside the film.

In this paper we present a study of the reflectivity of thin antiferromagnetic films discussing the case of oblique incidence of a $p$-polarized wave incident on an optically nonlinear film. The present work extends previous calculations where the transmission of a normally incident radiation was investigated. ${ }^{5}$ We note that, if nonlinear effects are considered, the frequency and power of the incident radiation are the key parameters to determine the optical behavior of the film.

In the following we develop a complete calculation of the reflectivity of uniaxial antiferromagnetic films of arbitrary thickness near resonance and at oblique incidence. We follow the calculation done by Almeida and Mills, ${ }^{5}$ making the necessary modifications to incorporate the effect of the incidence angle. Some years ago, Toussaint et al. ${ }^{11}$ reported on measurements of magnetic polaritons in $\mathrm{Mn}$-doped $\mathrm{FeF}_{2}$ samples whose antiferromagnetic resonance line width is less than $20 \mathrm{G}$. The resonance line

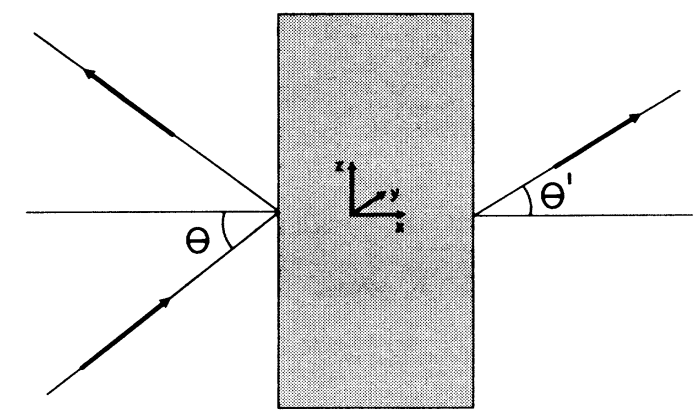

FIG. 1. A schematic illustration of the geometry used in the calculations. 
width poses a limit on the value of the incident radiation frequency for reflectivity measurements. However, it will be shown below for a $\mathrm{FeF}_{2}$ film that strong nonlinear effects can be induced if the incident radiation frequency is $150 \mathrm{G}$ below the antiferromagnetic resonance frequency if the thickness of the film is chosen conveniently.

The geometry used below is the following: we assume the film surface lies in the $y z$ plane and contains the an- isotropy axis which is parallel to the $z$ axis. The film surfaces are at $x=0$ and $x=d$ and the incident $r f$ magnetic field is polarized in the $y$ direction (see Fig. 1). The field equations and boundary conditions do not couple the polarizations and the solution everywhere is also a linearly polarized wave. Hence, we can write the magnetic field in the film as $\mathbf{H}=H(x, t) \hat{y}$ and the solution for Maxwell's equation is of the form:

$$
H=\exp (-i \Omega t)\left\{\begin{array}{l}
h_{i} \exp (i q x)\left[\exp \left(i k_{z} z\right)+R \exp \left(-i k_{z} z\right)\right], \quad \text { if } x>0 \\
\left.\mathcal{H}(x) \exp \left\{i \phi(x)+k_{z} z\right]\right\}, \quad \text { if } 0<x<d \\
T h_{i} \exp \left[\left(q x+k_{z} z\right), \quad \text { if } x<d\right.
\end{array}\right.
$$

where $k_{z}=\Omega \sin \theta / c, k_{x}=\Omega \cos \theta / c, \theta$ is the angle between the wave vector $\mathbf{k}$ of the incident wave and the $x$ axis, and $\mathscr{H}(x)$ and $\phi(x)$ are real functions. The field amplitude $\mathscr{H}(x)$ is determined by $\nabla^{2} H+\left(\epsilon \Omega^{2} / c^{2}\right) B=0$ with $\epsilon$ denoting the dielectric constant in the basal plane. The strength of the magnetic field induction $B(\mathbf{B}=B \hat{y})$ is given by $B=\left[\mu+12 \pi \chi_{0}(\Omega) \lambda(\Omega) H^{2}\right] H$, with $\chi_{0}(\Omega)$ denoting the linear part of the susceptibility, ${ }^{12}$ $\mu=1+4 \pi \chi_{0}(\Omega)$ and $\lambda(\Omega)$ representing the nonlinear content of the dynamical response. ${ }^{5}$

Writing the field in units of the incident magnetic field amplitude $\left(h=\mathcal{H} / h_{i}\right)$ we find $h(x)$ obeying the equation:

$$
\begin{array}{r}
\frac{d^{2}}{d x^{2}} h(x) e^{i \phi(x)}+k^{2}\left[\mu \epsilon-\sin ^{2} \theta+g(\Omega) h(x)^{2}\right] h(x) \\
\times e^{i \phi(x)}=0,
\end{array}
$$

where $g(\Omega)=12 \pi \epsilon \chi_{0}(\Omega) \lambda(\Omega) h_{i}^{2}$.

The solutions to Eq. (1) must satisfy the regular boundary conditions for the electromagnetic field on both surfaces of the film $(x=0$ and $x=d)$. The continuity of these field components at the surfaces leads to four equations which relate the magnetic field amplitude, phase, reflectivity, and transmissitivity of the film.

The real and imaginary parts of Eq. (1) give

$$
\frac{d^{2} h}{d x^{2}}-h\left(\frac{d \phi}{d x}\right)^{2}+\left(\mu \epsilon-\sin ^{2} \theta\right) k^{2} h+k^{2} g(\Omega) h^{3}=0
$$

and

$$
\frac{d}{d x}\left(h^{2} \frac{d \phi}{d x}\right]=0 \text {. }
$$

From (3) we can see that $d \phi / d x=\xi / h^{2}$, where $\xi$ is a constant proportional to the time average energy flux through the film. Using this result, Eq. (2) can be rewritten as

$$
\frac{d^{2} h}{d x^{2}}-\frac{\xi^{2}}{h^{3}}+k^{2}\left(\mu \epsilon-\sin ^{2} \theta\right) h+k^{2} g(\Omega) h^{3}=0
$$

which leads to a second constant of integration, $\tau$, given by

$$
\tau=\left[\frac{d h}{d x}\right]^{2}+\frac{\xi^{2}}{h^{2}}+k^{2}\left(\mu \epsilon-\sin ^{2} \theta\right) h^{2}+\frac{1}{2} k^{2} g(\Omega) h^{4} .
$$

Equation (5) can be integrated and the result is

$$
\begin{array}{r}
\int_{h(x)}^{h\left(x_{0}\right)} \frac{h d h}{\sqrt{\tau h^{2}-\xi^{2}-k^{2}\left(\mu \epsilon-\sin ^{2} \theta\right) h^{4}-\frac{1}{2} k^{2} g(\Omega) h^{6}}} \\
= \pm\left(x-x_{0}\right) .
\end{array}
$$

The continuity of the fields at $x=d$ leads to $(d \phi / d x)_{x=d}=q \epsilon$ and therefore $\xi=h^{2}(d)(d \phi / d x)_{x=d}$ $=T^{2} q \epsilon$.

On the other hand, the possible patterns of the field inside the film are restricted by the boundary conditions. It can be shown that

$$
h^{2}(0)=\left\{\left(\epsilon^{2} \cos ^{2} \theta-\delta\right)+\sqrt{\left(\delta-\epsilon^{2} \cos ^{2} \theta\right)-2 g(\Omega)\left[4 \epsilon^{2} \cos ^{2} \theta-\left(3 \epsilon^{2} \cos ^{2} \theta+\delta\right) T^{2}-g(\Omega) T^{4}\right]}\right\} / g(\Omega)
$$

where $\delta=\mu \epsilon-\sin ^{2} \theta$.

Writing the denominator of Eq. (6) as a third degree polynomial in the variable $h^{2}$, we obtain $h(x)$ in terms of Jacobian elliptic functions ${ }^{13}$

$$
h^{2}(x)=P^{2}+\left(T^{2}-P^{2}\right) c n^{2}\left(\sqrt{g(\Omega)\left(T^{2}+Q^{2}\right) / 2} k(x-d) \mid \frac{T^{2}-P^{2}}{T^{2}+Q^{2}}\right) \text { for } T^{2}>P^{2}
$$

and

$$
h^{2}(x)=-Q^{2}+\left(T^{2}+Q^{2}\right) d n^{-2}\left[\sqrt{g(\Omega)\left(P^{2}+Q^{2}\right) / 2} k(x-d) \mid \frac{P^{2}-T^{2}}{T^{2}+Q^{2}}\right] \text { for } T^{2}<P^{2}
$$

with $P^{2}$ and $Q^{2}$ given by

$$
P^{2}=-\frac{1}{2 g(\Omega)}\left\{2 \delta+g(\Omega) T^{2}-\sqrt{\left[g(\Omega) T^{2}+2 \delta\right]^{2}+8 \epsilon^{2} T^{2} g(\Omega) \cos ^{2} \theta}\right\}
$$


and

$$
Q^{2}=\frac{1}{2 g(\Omega)}\left\{2 \delta+g(\Omega) T^{2}+\sqrt{\left[g(\Omega) T^{2}+2 \delta\right]^{2}+8 \epsilon^{2} T^{2} g(\Omega) \cos ^{2} \theta}\right\} .
$$

Equations (7) - (10) provide a complete analytical solution to the problem. However, notice that the transmissivity $T^{2}$, yet to be determined, is a parameter in these equations. The numerical technique to solve Eqs. (7)-(10) consists in finding values of $T^{2}$ for which Eqs. (7) and (10) are satisfied simultaneously. These values of $T^{2}$ define the possible modes of radiation transmission through the film. Since $T^{2}$ is bounded, for given values of $d, h_{i}, \Omega$, and $\theta$, the search is restricted to the interval $[0,1]$. From the value of $T^{2}$, the reflectivity of the film can be obtained.

In Figs. 2(a)-2(d) we show the reflectivity (ratio of the reflected to the incident energy) of a $73.5 \mu \mathrm{m}$ thick $\mathrm{FeF}_{2}$ film, as a function of the strength of the incident field for four different values of the incident angle $\theta$. We have used the following characteristic parameters for this uniaxial antiferromagnetic material: the anisotropy field $H_{a}=200.0 \mathrm{kOe}$, the exchange field $H_{e}=540.0 \mathrm{kOe}$, and the saturation magnetization of each sublattice $M_{s}=0.56$ $\mathrm{kG}$. These values of the exchange and anisotropy field lead to a zero-field value of the resonance frequency of $1.4 \times 10^{12} \mathrm{~Hz}$ (infrared region).

Analyzing Figs. 2 we can conclude that the nonlinear optical effect is strongly dependent on the incident angle. Comparing Figs. 2(a) and 2(c) we can see that the minimum value of incident field strength to produce multistability decreases when the incident angle increases. For example, if $\theta=0^{\circ}$, the multistability appears for values of $h_{i}$ around $3.2 \mathrm{G}$ (incident power $\simeq 12.2$ $\mathrm{W} / \mathrm{mm}^{2}$ ) while less than half of this power is necessary to produce the same effect if $\theta=60^{\circ}$.

Another aspect of considerable interest, with regards to possible practical applications of antiferromagnetic films, is the control of the transmissivity. In a previous study, for incident radiation normal to the film surface, Almeida and Mills ${ }^{5}$ showed that the transmissivity exhibits a nonregular sequence of peaks (Figs. 1-3 of Ref. 5) as a function of incident radiation frequency, for a fixed incident power. The peaks correspond to the condition of total transmission $\left(T^{2}=1\right)$ and their distribution is far from the regular pattern exhibited in linear regime.

We address this question in a complementary way. Since the frequency of the incident radiation, the incident field amplitude and the angle of incidence are parameters at our disposal, we fix the incident radiation frequency and examine the transmissivity as a function of incident power and incidence angle. For a given value of the incident field amplitude, we search values of $\theta$ for which the transmitted power is nearly $1.0\left(T^{2}>0.99\right)$. In Figs. 3(a) and 3(b) we show, for frequencies of 150 and $300 \mathrm{G}$ below the resonance, the values of the incidence angle for which the reflected radiation is less than $1 \%$ of the incident power. We have used the same parameters of the film of Figs. 2 to obtain the results shown in Figs. 3. As can be seen in Figs. 2, for that frequency and thickness, in the limit $h_{i} \rightarrow 0$ (linear regime), less than $60 \%$ of the in-
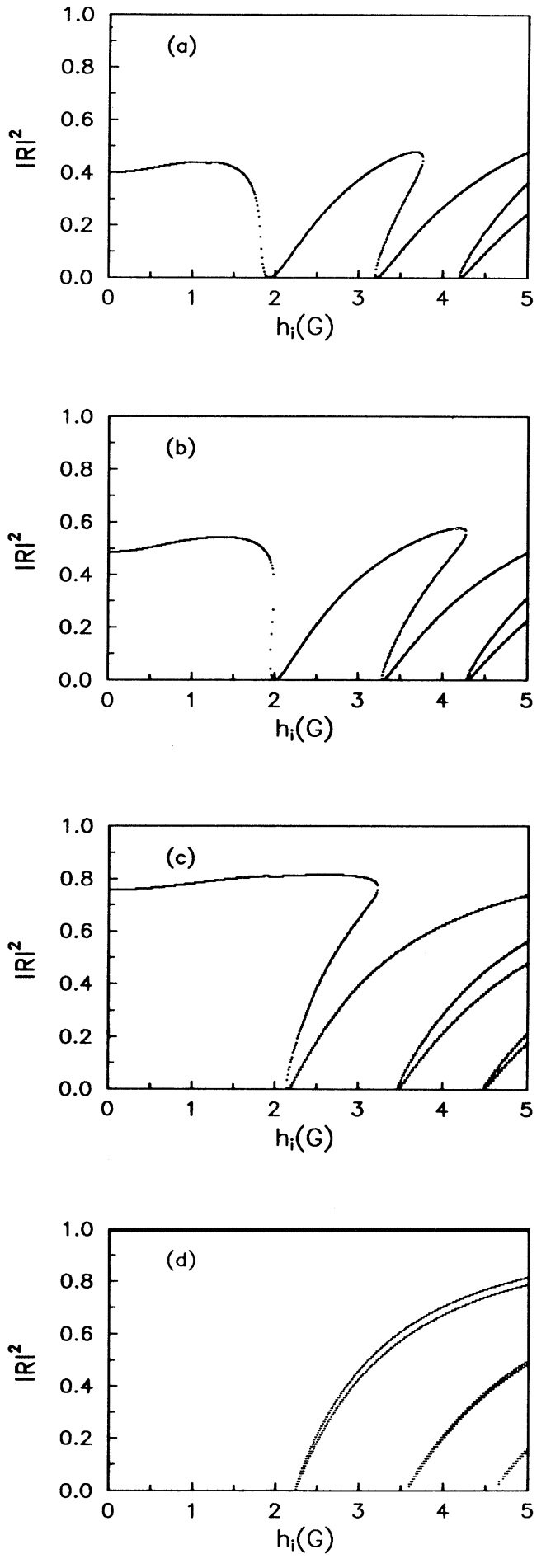

FIG. 2. Reflectivity of a $\mathrm{FeF}_{2}$ film, $85 \mu \mathrm{m}$ thick as a function of the strength of the incident radiation field for (a) $\theta=0^{\circ}$; (b) $\theta=30^{\circ}$; (c) $\theta=60^{\circ}$; (d) $\theta=85^{\circ}$, for incident radiation frequency $150 \mathrm{G}$ below the antiferromagnetic resonance frequency. 

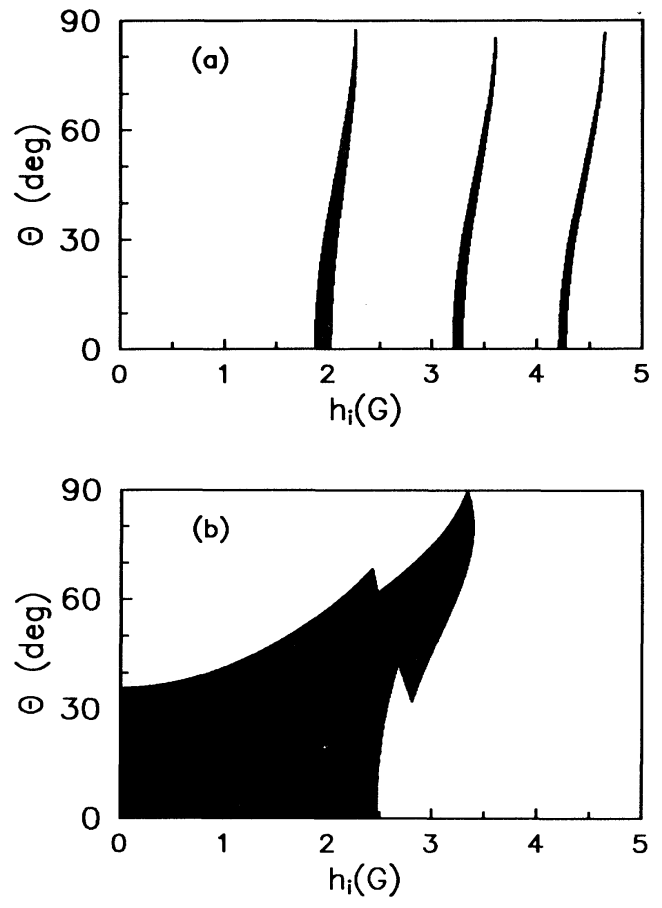

FIG. 3. Values of $\theta$ for which the radiation reflected by the film of Fig. 2 is less than $1 \%$ for different values of the strength of the incident field and (a) frequency $150 \mathrm{G}$ below to the resonance (b) frequency $300 \mathrm{G}$ below to the resonance.

cident power is transmitted through the film for any angle of incidence. When the incident power is increased, the nonlinear term of the susceptibility modifies the optical density of the film and the conditions for total transmission of incident power correspond to narrow intervals of the incident field. On the other hand, for the frequency used in Fig. 3(b), in the linear regime the incident power is almost totally transmitted for $\theta<34^{\circ}$. When the incident power is increased it can be seen that the first effect of the nonlinear term is a correction in the linear refraction index. This fact appears in Fig. 3(b) as an increase in the maximum angle for which the reflectivity is smaller than $1 \%$. Since the nonlinear term

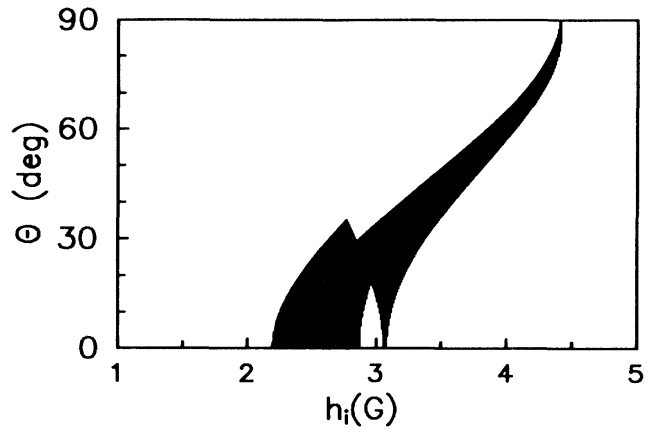

FIG. 4. Values of $\theta$ for which the radiation reflected by a 210 $\mu \mathrm{m} \mathrm{FeF}$ is less than $1 \%$ for different values of the strength of the incident field and frequency $300 \mathrm{G}$ below to the resonance.

depends on the frequency, the multistability appears only for fields higher than that of Fig. 3(a). In Fig. 4 we present results for a thicker film. The film is $210 \mu \mathrm{m}$ thick and we can see that there is a value of the incident field amplitude below which total transmission is not allowed $\left(h_{i}=2.2 \mathrm{G}\right)$. Just beyond this critical value, the nonlinear term produces modifications in the refractive index and multistability appears for $h_{i} \geq 2.9$. Narrow windows of total transmission appear for very high incident power. These results indicate that windows of total transmission can be tailored by choosing appropriate values of film width for a given incident radiation power.

We have presented a theoretical study of the nonlinear reflectivity of antiferromagnetic films. The calculations are based in a simple, but yet accurate, numerical technique that allows the study of the effects of incident radiation frequency and power, incident angle and film thickness on the reflectivity. We have shown that the incidence angle affects the reflectivity strongly. No comparison to experimental results is possible at this time. We hope the present work can motivate further experimental studies of radiation transmission through thin antiferromagnetic films.

This research was partially supported by the Brazilian Research Council (CNPq).
${ }^{1}$ H. M. Gibbs, Optical Bistability: Controlling Light with Light (Academic, New York, 1985).

${ }^{2}$ Y. R. Shen, Principles of Nonlinear Optics (Wiley, New York, 1984).

${ }^{3}$ Proceedings of the International Conference on Optical Nonlinearity and Bistability of Semiconductors [Phys. Status Solidi B 150, 349 (1988)].

${ }^{4}$ Th. Peschel, P. Dannberg, U. Langbein, and F. Lederer, Opt. Soc. Am. B5, 571 (1988).

${ }^{5}$ N. S. Almeida and D. L. Mills, Phys. Rev. B 36, 2015 (1987).

${ }^{6}$ L. Kahn, N. S. Almeida, and D. L. Mills, Phys. Rev. B 38, 8072 (1988).
${ }^{7}$ L. Kahn, K. Huang, and D. L. Mills, Phys. Rev. B 39, 12449 (1989).

${ }^{8}$ W. Chen and D. L. Mills, Phys. Rev. B 38, 12814 (1988).

${ }^{9}$ W. Chen and D. L. Mills, Phys. Rev. B 35, 524 (1987).

${ }^{10}$ N. S. Almeida and A. S. Carriço, Solid State Commun. 72, 181 (1989).

${ }^{11}$ R. M. Toussaint, D. W. Hone, V. Jacarino, and S. M. Rezende, Phys. Rev. B 38, 59 (1984).

${ }^{12}$ D. L. Mills and E. Burstein, Rep. Prog. Phys. 37, 817 (1974).

${ }^{13}$ L. M. Milne-Thomson, Jacobian Elliptical Function (Dover, New York, 1950). 